\title{
Psicologia Ambiental e Estudos PessoAs- AMBIENTE: QUE TIPO DE COLABORAÇÃO MULTIDISCIPLINAR?
}

\author{
Gabriel Moser \\ Universidade Paris $V$
}

\begin{abstract}
Este artigo procura definir quais as colaborações existentes entre a Psicologia Ambiental e os diversos estudos pessoas-ambiente a partir dos trabalhos inscritos no Congresso IAPS-16 (Paris, 2000). Na relação entre transdiciplinaridade, pluridisciplinaridade e interdisciplinaridade, entende que o funcionamento científico é necessariamente monodisciplinar, enquanto a intervenção nas relações pessoas-ambiente envolve uma dinâmica complexa. Quanto à intervenção (manejo sócioambiental), distingue dois tipos de funcionamento: interdisciplinar $e$ transdisciplinar. Conclui por um conhecimento que integre as diversas abordagens disciplinares em abordagens transdiciplinares.
\end{abstract}

Descritores: Psicologia Ambiental. Pesquisa interdisciplinar. Interdisciplinaridade. Transdisciplinaridade.

$\mathrm{U}$

$\mathrm{m}$ aspecto característico das relações pessoas-ambiente é que, em qualquer relação ambiental, temos de focalizar nossa atenção no usuário do ambiente tanto quanto no próprio ambiente. $\mathrm{O}$ ambiente é, por essência, um campo multidisciplinar, e as diferentes fontes de questionamento das diferentes disciplinas enriquecem as colaborações e trocas interdisciplinares. Diferentes níveis e questões não apresentam os mesmos desafios para as

1 Professor de Psicologia Ambiental e coordenador do programa de Especialização em Psicologia Ambiental da Universidade Paris V (França). Diretor do Laboratório de Psicologia Ambiental do Centro Nacional de Pesquisa Científica da França; membro da diretoria da IAPS. Endereço eletrônico: gabriel.moser@univ-paris5.fr

Psicologia USP, 2005, 16(1/2), 131-140 
profissões de design e planejamento e para os cientistas sociais. Suas perspectivas de análises e intervenções freqüentemente têm pontos de partida diferentes, focalizando ora o ambiente construído ora as pessoas nele vivendo. Há, também, algumas vezes, uma lacuna seqüencial: arquitetos, designers e planejadores intervêm no processo de concepção e construção de contextos nos quais as pessoas vivem, enquanto as ciências sociais geralmente dirigem a sua atenção para estruturas existentes. Lidar com as relações das pessoas ao ambiente global parece ser mais do domínio de intervenção da ciência social, mesmo se, de fato, dentro do International Asssociation for People-Environment Studies (IAPS) haja um forte comprometimento multidisciplinar entre os diversos domínios dos estudos pessoas-ambiente.

\section{Quem trata qual tipo de temática?}

As intervenções que se referem principalmente ao habitat e design de espaços coletivos estão baseadas em colaborações multidisciplinares entre arquitetos, designers e cientistas humanos. O manejo ("engineering") sócioambiental freqüentemente implementa a lógica transdisciplinar na solução do problema. Estratégias de intervenção referentes ao ambiente global são, na maior parte dos casos, intervenções monodisciplinares que levam à proposição de soluções em um enquadramento de identificação multidisciplinar do problema.

A análise de conteúdo das diferentes categorias de trabalhos submetidos ao Congresso IAPS 16 (Conference of International Association for People - Environment Studies), realizado em Paris em 2000, proporcionam uma visão panorâmica e de perspectivas quanto à pesquisa atual internacional em pessoas-ambiente. As apresentações cobrem a seguinte temática (cada uma das quais com cerca de 100 trabalhos).

1. Sustentabilidade e comportamento ecológico. Esta temática inclui uma perspectiva teórica sobre sustentabilidade, política ambiental, questões de poluição ambiental, a relação das pessoas com a natureza e com os recursos naturais; preocupação ecológica. 
2. Comunidade, identidade e cultura. A pesquisa nesta seção inclui participação e implicação, diversidade urbana, apego e identidade ao lugar, satisfação residential e qualidade de vida, vida no subúrbio e modo urbano de vida, crianças e ambiente

3. Habitação e espaço proximal. Esta inclui moradia e vizinhança, moradias precárias, design de interiores, vários aspectos de ambientes específicos (escritórios, ambientes para o turismo e para o lazer, para os idosos, etc.).

4. Mudança urbana. Esses tópicos incluem cidades em encruzilhadas (cidades em transformação, cidades em crescimento, transformações urbanas); políticas urbanas; em direção de ambientes urbanos sustentáveis, espaços públicos, parques urbanos, lugares de história e memória.

As questões clássicas de estudos pessoa-amiente (habitação, bairro, estudos urbanos) são principalmente monodisciplinares, conduzidas seja por arquitetos e designers, seja por cientistas sociais. Os interesses em comunidade, identidade e cultura são conduzidos por, e em igual quantidade, tanto por profissionais de design quanto por cientistas sociais. Por outro lado, a preocupação com o ambiente global e o comportamento ecológico envolve, principalmente, somente uma única abordagem disciplinar, neste caso, as ciências sociais, com a identificação do problema originado-se muitas vezes de várias disciplinas tradicionalmente não implicadas em estudos pessoaambiente, tais como economia e biologia.

\section{Como a abordagem interdisciplinar se expressa?}

Examinando as 98 sessões do IAPS 16, a maioria delas combina intervenções de diferentes disciplinas em um mesmo tópico, proporcionando, assim, uma oportunidade para uma discussão multidisciplinar. Isto significa que o lugar e a proporção de abordagens multidisciplinares de um mesmo 
tópico é altamente promissor. De fato, em mais de três quartos de todas as sessões (78\%), há trabalhos apresentados por cientistas sociais tanto quanto por arquitetos, planejadores e designers. Isto significa que as discussões interdisciplinares são bastante freqüentes. Mesmo na temática de mudança urbana, onde a proporção de ciências sociais versus arquitetos, planejadores e designers é mais baixa, somente $30 \%$ das sessões são compostas por trabalhos reunindo apenas arquitetos, planejadores e designers ou cientistas sociais.

No total, aproximadamente dois terços dos trabalhos (62\%) são apresentados por arquitetos, planejadores e designers, mas esta proporção não está igualmente distribuída em todas as categorias temáticas. Especialmente os trabalhos tratando de "Habitação e espaço proximal" e de "Mudança urbana" são significativamente mais apresentados por arquitetos, planejadores e designers do que por cientistas sociais.

Figura 1: Porcentagem da principal disciplina envolvida por categoria temática

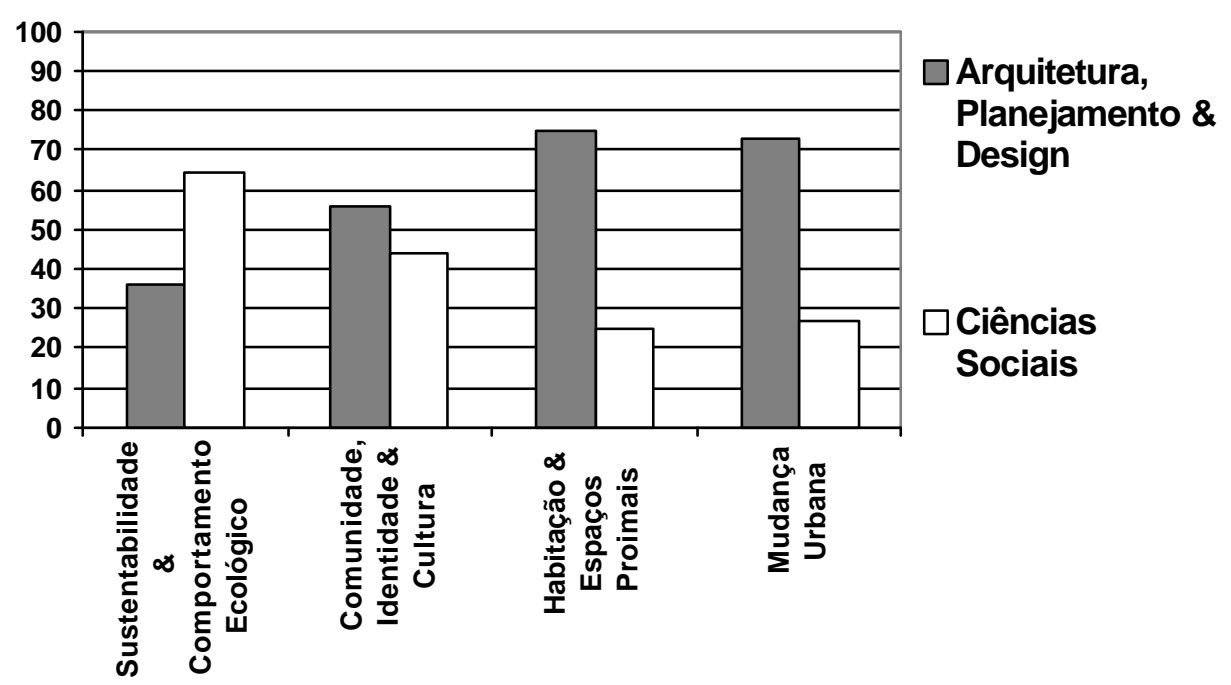

A figura 1, referente aos interesses de pesquisa dos membros da IAPS, reflete bastante adequadamente a orientação e prioridades atuais da pesquisa internacional ambiente-comportamento. Representa claramente um incenti- 
vo para as ciências sociais intervirem mais em pesquisa e prática que lidem com habitação, espaços proximais e estudos urbanos.

\section{Pluridisciplinaridade, interdisciplinaridade e transdisciplinaridade: da pesquisa à intervenção}

Na Psicologia Ambiental, não há uma distinção precisa entre a pesquisa básica e a aplicada. A aplicabilidade da pesquisa é central às questões pessoas-ambiente. A característica da pesquisa e da prática pessoasambiente é o equilíbrio entre a monodisciplinaridade, que é necessária para a pesquisa e para a construção de teoria nas diferentes disciplinas implicadas nas questões pessoas-ambiente, e a multi ou inter-disciplinaridade (confrontação e/ou colaboração com outras disciplinas), indispensável para a intervenção ambiental.

A Psicologia Ambiental funciona de um modo indutivo e orientadoao-problema. Mesmo quando as definições dos problemas são multidisciplinares, a pesquisa e a construção de teoria requerem abordagens centradasna-disciplina que são, necessariamente, mono-disciplinares, produzindo um conhecimento disciplinar referido a conceitos disciplinares. A pesquisa e a construção da teoria estão baseadas nos paradigmas principais específicos à disciplina e, deste modo, podem apenas se referir aos conceitos específicos à disciplina.

Enquanto que o funcionamento científico é necessariamente monodisciplinar, a intervenção nas relações pessoas-ambiente, ao contrário, envolve uma dinâmica complexa. Reponder demandas sociais freqüentemente combina múltiplas abordagens envolvendo diferentes disciplinas, cada uma delas com as suas próprias características, seguindo a sua própria lógica disciplinar. Apenas a colaboração com outras disciplinas comprometidas com questões pessoas-ambiente garante uma solução eficiente a questões ambientais.

As modalidades de intervenção e o recurso a outras disciplinas dependem do momento do uso da colaboração no processo de intervenção (diag- 
nóstico / intervenção / avaliação e controle da adequação das transformações implementada), e da natureza da distribuição das tarefas alocadas às diferentes disciplinas envolvidas (cada disciplina participando igualmente de cada estágio, ou uma das disciplinas operando como consultor para outra disciplina envolvida no processo). Os diferentes tipos de colaborações entre disciplinas nas questões ambientais podem ser resumidas como se segue (Quadro 1)

Quadro 1 Objetivos e colaboração entre disciplinas

Pesquisa e

Monodisciplinaridade (conhecimento disciplinar)

construção de teoria

Diagnóstico/exame

Multidisciplinaridade (múltiplas lógicas disciplinares)

Intervenção

Interdisciplinaridade (confrontação e/ou colaboração em torno de uma problemática comum)

Transdisciplinaridade (Identificação de problemas, análises integradas e respostas em comum)

Com respeito à intervenção (manejo sócio-ambiental), dois tipos diferentes de funcionamento podem ser distinguidos (Quadro 2):

- Funcionamento interdisciplinar: consiste em uma abordagem múltipla e paralela de um mesmo objeto ou problema. A abordagem implementada tem recorrido a diferentes disciplinas que intervêm com sua lógica específica para analisar o tópico. Cada disciplina proporciona uma solução com referência à sua própria lógica científica. Mesmo se as soluções possíveis forem específicas a uma disciplina, as confrontações interdisciplinares no estágio final garatem a pertinência ecológica da proposição.

- Transdisciplinaridade: é uma abordagem que incorpora as diferentes disciplinas em colaboração em todos os estágios da solução do problema. A 
definição do propósito da intervenção, as estratégias implementadas e as recomendações são fundamentadas em uma abordagem comum da problemática e levam a proposições integradas das soluções possíveis. Enquanto que a transdisciplinaridade ocorre com facilidade quando referida a disciplinas tendo o mesmo tipo de abordagem, i.e., dentro das Ciências Sociais ou dentro das profissões de design, por causa de uma racionalidade comum no modo de olhar os problemas, a colaboração entre as Ciências Sociais e as profissões de design é mais complexa e leva, freqüentemente, na prática, a um recuo defensivo para uma abordagem interdisciplinar, mais confortável, do problema.

Quadro 2 Tipos de funcionamento com respeito à intervenção

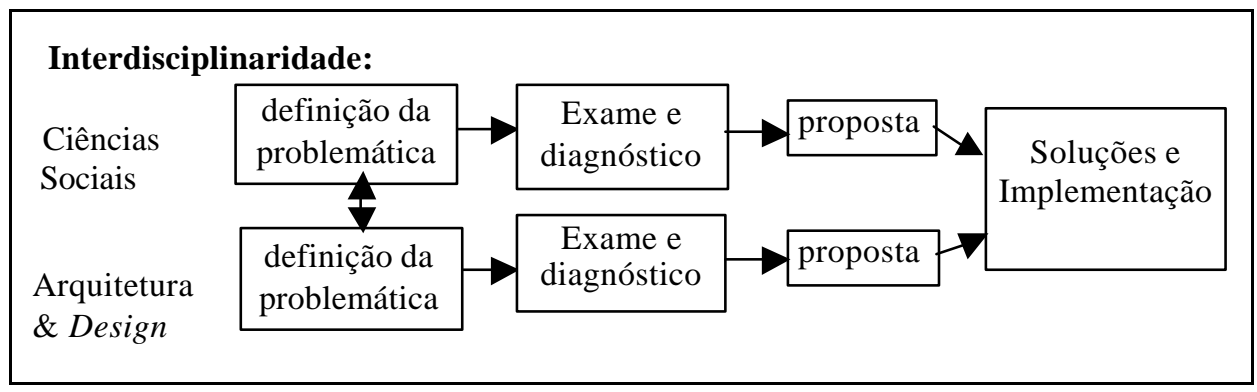

\section{Transdisciplinaridade:}

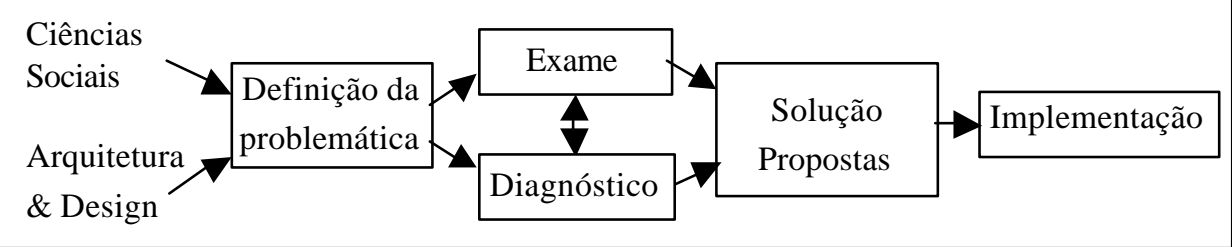

Duas modalidades de transdisciplinaridade podem ser distinguidas quanto aos tipos de disciplinas envolvidas:

1. Colaboração com outras Ciências Sociais: O objeto de estudo do psicólogo ambiental não é específico a uma disciplina. Outras disciplinas provavelmente vão se reconhecer no mesmo objeto. Mais 
especificadamente, as ciências humanas e sociais, como a geografia, a ecologia, a sociologia, são muito próximas da psicologia ambiental em sua concepção das relações humano-ambiente no sentido de que o seu ponto de partida é o indivíduo em estudos pessoas-ambiente. A colaboração com disciplinas relacionadas, referidas a uma mesma racionalidade (rational), não competem quanto aos paradigmas fundamentais. Em tais casos, a implementação das abordagens trandisciplinares são mais fáceis e, assim, também mais freqüientes.

2. Colaboração com as profissões do design: A solução de problemas transdiciplinares em colaboração com arquitetos e designers é mais complexa, pois os dois grupos de disciplinas têm um ponto de partida diferente, focalizando seja o ambiente construído ou como as pessoas vivem nele. Implica que a problemática é reformulada de um modo a integrar racionalidades diferentes e para permitir uma operacionalização comum. Tais condições ideais para a abordagem transdiciplinar são raramente atingidas. A intervenção raramente integra diferentes disciplinas de um modo que permita construir uma definição e abordagem, específica e única, do objeto.

\section{Conclusões: Desenvolvimento sustentável e multidisciplinaridade}

O conhecimento acumulado durante o último século proporciona condições ótimas para responder aos requisitos de sustentabilidade neste novo século. Para manter a produção neste alto nível de conhecimento e de expertise, e para contribuir significativamente aos desafios deste novo século, os especialistas pessoas-ambiente terão de alcançar duas metas:

1. comprometer-se crescentemente com abordagens multiculturais e transculturais, dentro de uma perspectiva histórica, com respeito ao ambiente construído a fim de introduzir a dimensão temporal no estudo das relações pessoas-ambiente; 
2. comprometer-se crescentemente com abordagens transdisciplinares dos diferentes temas clássicos, combinando as contribuições dos planejadores urbanos, arquitetos, designers e psicólogos ou sociólogos, a fim de garantir a validade ecológica ótima e a aplicabilidade dos resultados das pesquisas.

Apenas o conhecimento que integre as diversas abordagens disciplinares, seja pelo confronto ou pelo entrosamento em abordagens transdiciplinares, proporcionará uma resposta adequada aos desafios deste novo século. Apenas a colaboração entre as ciências sociais e as disciplinas de design pode garantir uma resolução bem sucedida dos problemas em questões ambientais. O IAPS parece ser o lugar onde um tal intercâmbio produtivo entre disciplinas pode acontecer.

Moser, G. (2005). Environmental psychology and people-environment studies: What kind of multidisciplinary collaboration? Psicologia USP, 16(1/2), 131-140.

\begin{abstract}
This article aims to show the existing collaborations between Environmental Psychology and the diverse people-environment studies, based on the works presented in the Congress IAPS-16 (Paris, 2000). In the relation between transdisciplinarity, pluridisciplinarity and interdisciplinarity, it understands that scientific functioning is necessarily mono-disciplinary, while intervention in people-environment relations involves a complex dynamics. As for intervention (socio-environmental handling), it distinguishes two types of functioning: interdisciplinary and transdisciplinary. It concludes that only the knowledge that integrates the several disciplinary approaches into transdisciplinary approaches will provide an adequate answer to the challenges of this new century.
\end{abstract}

Index terms: Environmental psychology. Interdisciplinary research. Interdisciplinarity. Transdisciplinarity. 
Moser, G. (2005). Psychologie environnementale et etudes personnes environnement: quel type de collaboration multidisciplinaire? Psicologia USP, 16(1/2), 131-140.

Résumé: Cet article cherche à définir quelles sont les collaborations existantes entre la Psychologie Environmentale et les différentes études personnes -environnement.à partir des travaux inscrits au Congrès IAPS-16 (Paris, 2000). Dans la relation entre transdisciplinarité, pluridisciplinarité et interdisciplinarité, il estime que le fonctionnement scientifique est nécessairement monodisciplinaire, tandis que l'intervention dans les relations personnes-environnement implique une dynamique complexe. Quant à l'intervention (maniement socio-environnemental), il différencie deux types de fonctionnement: l'interdisciplinaire et le transdisciplinaire. Il en conclut que ce n'est que la connaissance qui intègre les différentes optiques disciplinaires dans des optiques transdisciplinaires qui fournira une réponse adéquate aux défis de ce nouveau siècle.

Mots-clés: Psychologie de l'environnement. Recherche interdisciplinaire. Interdisciplinaire. Transdisciplinaires.

\section{Referência}

Conference of the Internacional Association for People - Environment Studies, 16, Paris, 2000. (2000). Paris: IAPS.

Recebido em 5.04.2004

Revisto e encaminhado em 23.02.2005

Aceito em: 7.03.2005 